\title{
Statistical methods for decision making and risk measures
}

Techniques in applied statistics play an important role in decision making and risk measures. Many practical models are inspired by empirical studies and are developed to explain observed phenomena. This issue has collected six papers devoted to this theme. The papers have been presented in the International Conference on Applied Statistics and Financial Mathematics (ASFM2010) and International Research Forum on the current global economic crisis held in December 2010 in Hong Kong, P. R. China.

In the paper "A heuristic method for estimating attribute importance by measuring choice time in a ranking task" by Barone, Lombardo and Tarantino, a new practical method for indirectly capturing consumers' attribute is presented. It allows analysts to indirectly obtain a respondent's relative importance weights for several tested attributes through a simple and fast procedure. The method also makes it possible to overcome most of the problems related to context, survey and cognitive variables. This makes the new method more reliable than any of the existing alternatives, such as direct rating.

In the paper "Least squares estimators for nearly unstable processes for functionals of long-memory noises" by Chan and Liu, the asymptotic theory of the least squares estimators (LSE) for long-memory nearly unstable model when the innovation sequences are functionals of moving averages is investigated. It is shown that the limit distribution of the LSE is a functional of the Hermite Ornstein-Uhlenbeck process.

In the paper "Frailties and copulas, not two of a kind" by Goethals, Janssen and Duchateau, a unified framework is proposed for the copula model and the different types of frailty models in order to evaluate similarities and differences between the models. The frailty effect at the event time scale is investigated. When frailties operate at the hazard level and influence the event times in a nonlinear way, it leads in some instances to counterintuitive findings.
In the paper "A study on the effects of skewed distributions on the performances of max-EWMA and maxGWMA charts" by Teh and Khoo, a comparative study is carried out for the max-GWMA (called the maximum generally weighted moving average) chart and the max-EWMA (called the maximum exponentially weighted moving average) chart for simultaneously monitoring the process mean and/or variability. These charts find applications in statistical process control. Overall, the results show that the max-GWMA chart has lower false alarm rates for more levels of skewnesses, compared to the max-EWMA chart.

In the paper "Conditional moments, sub-independence and independence II" by Hamedani and Volkmer, a concept called sub-independence, which is defined in terms of the convolution of the distributions of random variables, is reviewed. The concept of subindependence allows the determination of the distribution of the sum of the components in a stochastic model based on the marginal distributions of the summands. This concept is much weaker than that of independence and yet can be employed to determine the distribution of the sum of random variables from their marginal distributions.

In the paper "A decomposition method for optimal portfolios with regime-switching and risk constraint" by Liu, Yiu and Siu, an optimal investment problem for an investor who faces a dynamic risk constraint in a Markovian regime-switching environment is studied. The goal of the investor is to maximize the expected utility of terminal wealth subject to the dynamic risk constraint specified by a proportional value-at-risk (VaR). By transforming the stochastic optimal control problem associated with the optimal investment problem into a deterministic control problem, a closed-form solution to the optimal investment problem for the case of a power utility is obtained. 